Victoriya Gnitsevych, Doctor of Technical Sciences, Professor, Kyiv National University of Trade and Economics, 19, Kyoto st., Kyiv, 02156, Ukraine,

ORCID: 0000-0002-6089-1082

ResearcherID: N-3463-2016

Olena Vasylieva,

$\mathrm{PhD}$ in Engineering Sciences Kyiv National University of Trade and Economics, 19, Kyoto st., Kyiv, 02156, Ukraine, ORCID: 0000-0002-1707-4546

ResearcherID: N-3357-2016

\title{
PROSPECTS OF USING LOCAL PLANT RAW MATERIALS IN THE TECHNOLOGY OF THE SEMI-FINISHED PRODUCTS FOR DESSERTS
}

The article is presenting theoretical background and experimental studies to determine the possibility of using local plant raw materials as a structure-forming component for the dessert production.

Keywords: plant raw materials, semi-finished products, structure-forming properties, desserts.

The relevance of the researched topic. There is a wide usage of foaming agents and emulsifiers in the restaurant industry. These components form the structural and mechanical properties of the finished product and determine its organoleptic characteristics. The usage of the natural plant materials for structure formation allows not only to expand the range of food products but also to give up the chemical food additives and rational use of local resources.

The usage of various surfactants in the composition of raw plant materials as structure-forming agents causes great interest. They have significant advantages over artificial additives and their mixtures because their valuable components are in the form of natural compounds and are better absorbed by the human body.

Problem setting. In the modern world of nutritional development, the creation of foodstuffs with specific properties is particularly important. Due to a large amount of biologically active compounds in them, natural additives from plant raw materials enhance the biological value and preventive properties of the finished product. The use of natural wild raw materials, which has been widely distributed in Ukraine, for the abovementioned purpose, allows not only improving the quality and expanding the range of products but also to the rational use of local resources [1]. 
Due to the features of chemical composition and technological properties, the processed products of plant raw material play a special role in the product technologies with foam and emulsion structure. Based on technological positions, in food foams and emulsions, plant additives with certain surface-active properties participate in the formation of such structures, hence allowing replacing traditional foaming agents and emulsifiers [2].

The basis for use of additives derived from plant raw materials for nutritional purposes should be based on detailed technological research, which will evaluate the behavioral mechanism during the process of dessert product preparation and determine the conditions and value of their use.

Analysis of recent research and publications. Today there is a whole area in the food industry directed on production and usage of foodstuffs, which are made of plant raw materials. Most of them are developed to improve the nutritional value; access to the general public; the rational use of local plant raw materials; expand the range of food repertoire for children, diet and therapeutic products [3-8].

Task setting. In this context, the plant's raw material looks especially promising. It is rich in biologically active substances and biopolymers, which allows increasing the nutritional value of products and reducing calories by enriching them with vitamins, minerals. Such raw materials include Jerusalem artichoke and amaranth. However, the existing technologies of their processing involve the extraction of biologically active substances (BAS) or a separate component (juices, concentrates, grist), which are mainly used as dietary supplements in technologies of special-purpose products. Scientific and applied researches aimed at the realization of the whole complex of functional and technological properties of the specified raw material are currently limited in Ukraine.

Presentation of the main material. The purpose of the work was to demonstrate the possibility of using plant raw materials as structure-forming agents in technologies of foam or emulsion structured products and to identify technological factors that influence the structure-forming processes.

General experience of scientific and practical researches allowed creating a scientific base for experimental researches, which is presented in Figure 1.

According to the schema, in the first stage, we have conducted a study, which was aimed to determine the role of the chemical composition of juices and purees of selected plant products (green amaranth, Jerusalem artichoke, Cornelian cherry dogwood) in the foaming and emulsification processes. Analysis of the chemical composition and studies has shown that the main structure-forming components of raw materials are protein substances, pectin, inulin, and saponin. The summary of the obtained experimental data, which characterize the foaming and emulsification indicators selected for studying plant juices and purees, is presented in Table 1. 


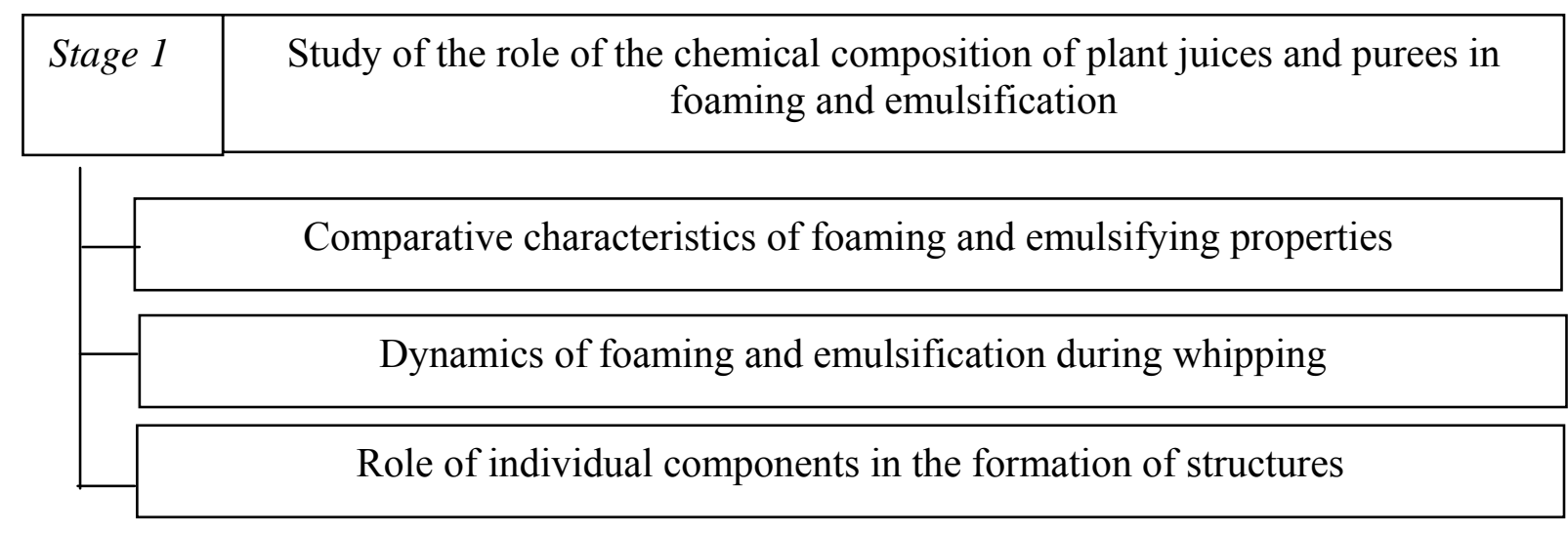

\begin{tabular}{|l|l|}
\hline Stage 2 & Study of foaming and emulsifying properties of model surfactant systems \\
\hline
\end{tabular}
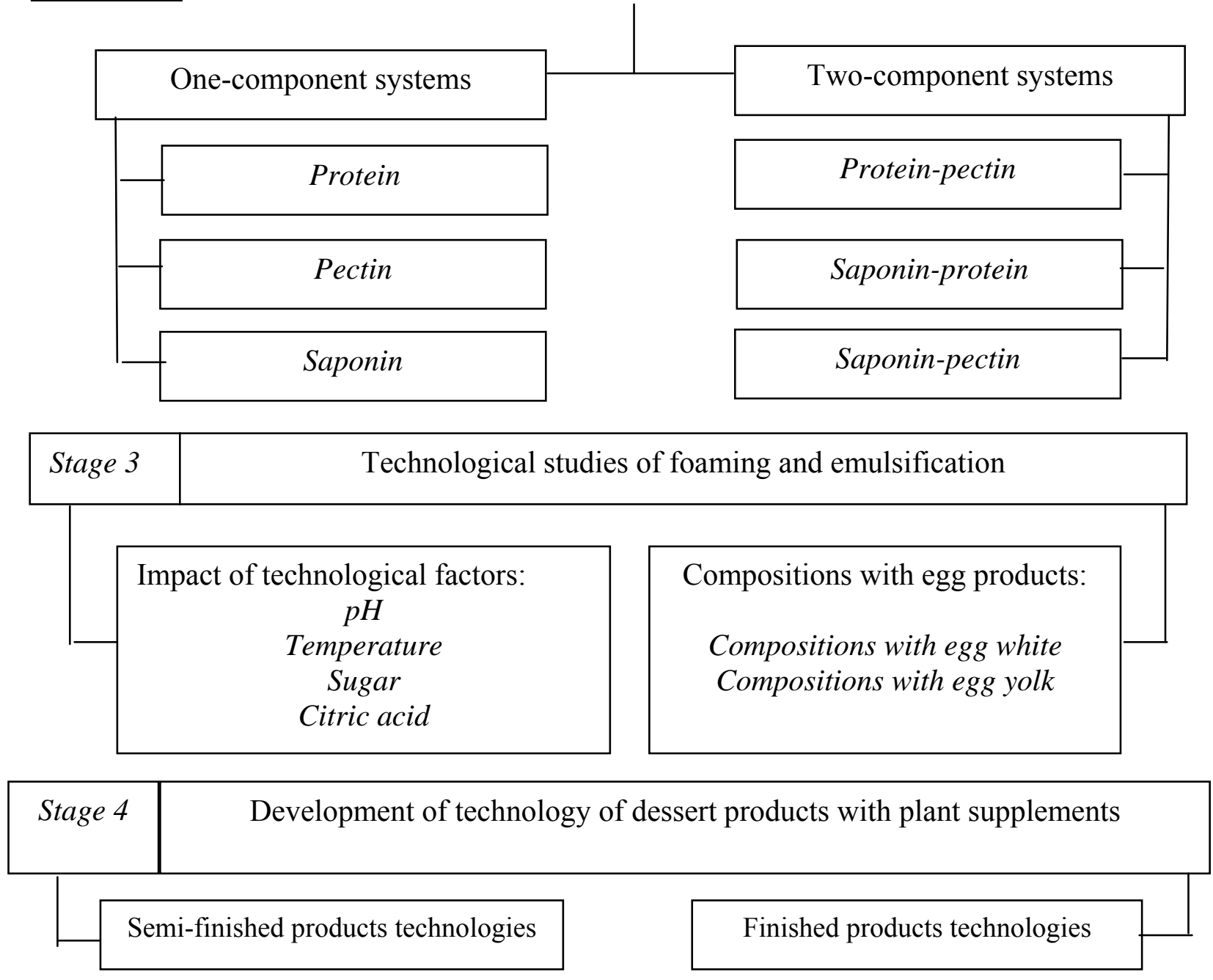

Figure 1. Schema of the experiment 
Comparative characteristics of the foaming and emulsifying properties of juices and purees provided a reason to claim that plant-derived juices have foaming ability, however, plant-derived purees do not form foam in their pure form but have pronounced emulsifying properties. It is obvious that such differences in the properties of additives from one raw material are related to their structural and mechanical properties since the numbers of surfactants in them are almost at the same level.

Table 1

\section{Foaming and emulsifying properties of juices and purees}

\begin{tabular}{|c|c|c|c|c|c|c|}
\hline \multirow{2}{*}{ Indicators } & \multicolumn{2}{|c|}{ Amaranth } & \multicolumn{2}{c|}{ Jerusalem artichoke } & \multicolumn{2}{c|}{$\begin{array}{c}\text { Cornellian cherry } \\
\text { dogwood }\end{array}$} \\
\cline { 2 - 7 } $\mathrm{pH}$ & Juice & Puree & Juice & Puree & Juice & Puree \\
\hline & $6.42 \pm 0.05$ & $5.82 \pm 0.06$ & $6.24 \pm 0.05$ & $5.62 \pm 0.06$ & $4.15 \pm 0.05$ & $\begin{array}{c}3.72 \pm \\
0.05\end{array}$ \\
\hline Surface tension, mN/m & $51.5 \pm 1.5$ & - & $42.2 \pm 1.6$ & - & $48.5 \pm 1.3$ & - \\
\hline The relative viscosity & $1.84 \pm 0.12$ & - & $1.32 \pm 0.11$ & - & $1.27 \pm 0.11$ & - \\
\hline $\begin{array}{c}\text { Effective viscosity, } \\
\mathrm{Pa} \bullet \mathrm{s}\end{array}$ & $1.1 \pm 0.05$ & $18.5 \pm 0.93$ & $1.0 \pm 0.05$ & $15.4 \pm 0.5$ & $1.0 \pm 0.04$ & $\begin{array}{c}14.3 \pm \\
0.85\end{array}$ \\
\hline Foaming ability, mm & $218 \pm 18$ & - & $258 \pm 10$ & - & $236 \pm 12$ & - \\
\hline Foam stability, \% & $42.3 \pm 3.4$ & - & $24.6 \pm 2.1$ & - & $27.7 \pm 2.3$ & - \\
\hline $\begin{array}{c}\text { Emulsifying ability, } \\
\mathrm{mm}\end{array}$ & $63 \pm 4$ & $75 \pm 4$ & $50 \pm 4$ & $62 \pm 5$ & $45 \pm 4$ & $57 \pm 4$ \\
\hline Emulsion stability, \% & $79.8 \pm 6.3$ & $84.6 \pm 6.6$ & $75.7 \pm 6.1$ & $81.6 \pm 6.5$ & $75.9 \pm 6.4$ & $80.7 \pm 6.7$ \\
\hline
\end{tabular}

Analysis of the dynamics of foaming and emulsification using juices and purees allowed us to make the following conclusions:

- The relative viscosity of juices decreases after they undergo the processes of foam and/or emulsion formation, which indicates the transition of those chemical components that have the properties of hydrocolloids to the formed structures (i. e. foams and/or emulsions).

- The increase of the surface tension and the weakening of the interphase adsorption layer at the boundaries of the phase separation imply the transitioning of raw surfactants into the foams and emulsions.

- The shift of active acidity towards neutral $\mathrm{pH}$ suggests the transition to foams and emulsions of the acidic components.

The result of such an interphase transition is a gradual decrease of the structureforming properties and the stability of the newly formed foams and emulsions.

Functional analysis of the raw material components in the processes of foaming and emulsification showed that amaranth has the foremost structure-forming properties since it consists of saponins, which in combination with proteins give the maximum 
effect; in Jerusalem artichokes, such effect is driven by proteins in combination with low- and high-polymeric fructans; in the Cornelian cherry dogwood, such effect is driven by proteins in combination with pectic substances. Studies of foaming and emulsifying properties of surfactants have allowed determining their rational relationships.

Since the use of plant supplements saves traditional foaming agents and emulsifiers in food technology, we consider that it is worth studying the foaming properties of egg white and egg yolk compositions with vegetable and fruit juices and purees.

It has been experimentally found that the foaming properties of egg white in combination with juices will increase, if the concentration of juices does not exceed $15-20 \%$, except for Jerusalem artichoke puree, where the amount of the additive can reach $25 \%$. The optimal emulsifying condition of puree in combination with egg yolk reached when the number of the additive is from 17 to $28 \%$. The maximum effect is reached when egg yolk is combined with an amaranth puree. The stability of the foams and emulsions of these compositions is slightly increasing, while an increase of the additives (i. e. juice and/or puree) in these compositions correlates with a decrease of the structural stability.

For this reason, the next stage was directed on studying the general pattern change of foaming and emulsifying properties of compositions under the influence of various technological factors and determining their whipping conditions. Based on the study results, the following conclusions have been made:

- Food systems, which contain amaranth or Jerusalem artichoke juice, show foaming and emulsifying properties in the $\mathrm{pH}$ range from 4 to 8 , in systems with Cornelian cherry dogwood these properties are reached at the $\mathrm{pH}$ range of 5-7, which can be explained by the presence of saponins in amaranth and inulin in Jerusalem artichoke.

- The ability to foam and emulsify juices with increasing temperature in the range from 20 to $60^{\circ} \mathrm{C}$ remains high, but obtained foams and emulsions are much less stable.

- The limit of sugar mass fraction for studied juice systems is on average from 15 to $20 \%$; the decrease of its concentration stabilizes the foams and emulsions; the increase of its concentration, on the contrary, worsens their stability.

Therefore, from the technological point of view, the expediency of using purees and juices of plant products is proven; the optimal conditions of their structure formation are determined. Furthermore, this allowed developing several technologies for semi-finished and dessert products [9-11].

During studies, has been proven advantages of the developed semi-finished products, and products based on them, over the traditional dessert products based on their nutritional and biological values. These values include calorie reduction; enrichment with non-starchy polysaccharides (i. e. inulin, fiber, pectin) and minerals (i. e. calcium, iron); the improved balance of amino acid index, «Ca:P» and «Ca:Mg» ratios; as well as increasing the digestibility by $10-15 \%$ for all products, which fully 
aligned with the current state concept of Ukraine of the healthy nutrition population. The complex qualitative indicators of the new semi-finished products are equal to 0.996-1.056, which corresponds to the «excellent» rating.

The optimal storage conditions of semi-finished products are substantiated; the regularities of quality control based on changes in the main indicators are established. It has been proven that organoleptic and microbiological parameters are stable at determined parameters during the recommended shelf life.

Conclusions. The complex of theoretical and experimental studies allowed substantiating the possibility of using purees and juices from amaranth, Jerusalem artichoke, and Cornelian cherry dogwood, as the structure-forming agents. Moreover, this also allowed us to establish optimal conditions for the technological process where they show maximum surfactant properties. Technologies of semi-finished and dessert products have been developed; their advantages over traditional products have been proven.

\section{REFERENCES}

1. Smolyar B. (2010) The main trends in the nutrition of the population of Ukraine [Nutrition problems ] - №2. - P. 5-9.

2. Rybak O. Some aspects of the formation of emulsions and foams in food industry / O. Rybak // Ukrainian Journal of Food Science. - 2013. - Vol.1. - № 1. P. 41-47.

3. Simakhina G. (2013) Substantiation of the choice of wild berries for freshfrozen semi-finished products [Scientific papers of the National University of Food Technologies]. - № 52. - P. 75-81.

4. A new direction of deep processing of raw materials: monografyya / R. Pavlyuk, V. Pogarska, L. Radchenko, V. Pavlyuk. - 2017. - P. 380.

5. Gnitsevich, V. (2014) Technology of food products with given properties on the basis of secondary dairy and vegetable raw materials: monograph / Donetsk: D., $337 \mathrm{p}$.

6. Kaprelyants L., Iorgachova K. (2003). Functional food products: monograph. - Od. : Fact, 312 p. [in Ukrainian].

7. Mazaraki A., Kravchenko M., (2012). Technology of food products of functional purpose: monograph. - K. : KNUTE. $-1116 \mathrm{p}$.

8. Sidorenko O. (2006). Formation of assortment and quality of fish products: monograph. $-\mathrm{K}$.: KNUTE $-112 \mathrm{p}$.

9. Gnitsevich, V. (2011). Development of technology for the production of semi-finished products based on dogwood Gnitsevich, / V. Gnitsevich, Vasilieva O., [Equipment and technologies of food production] Topic. zb. science. - № 27. P. 111-115

10. Gnitsevich V. Vasilieva O. (2015). Substantiation of parameters of production of whipped desserts on the basis of Jerusalem artichoke and dogwood semi-finished product [Scientific Bulletin of Poltava University of Economics and Trade. Series: Technical Sciences]. PUET № 1 (73). - P. 11-18. 